\title{
AN EFFECTIVE NUMERICAL SOLUTION OF GUIDED WAVES EQUATIONS
}

\author{
C.V. AVILOFF
}

Acoustical Institute of Academy of Sciences USSR, Shvernik st. 4, SU-117036 Moscow, USSR

\begin{abstract}
Resume - Le probleme aux limites pour un guide d"ondes bidimensionnel scalaire dont la vitesse de son et 1 a densite dependent lentement de $x$ et arbitrairement de $z$ est resalu par la methode de factorization additive. L"equivalence entre cette méthode et la solution monodirectionnelle aux modes couples est prouvéy. L"approprie probleme Cauchy pseudodifferentiel est resolu avec la precision donnée par un algorithme fonde sur 1 approximation Fadé de grand ordre. Le rendenent d"une realization programme, adapté pour les calculations de son sous-marin est discute.

Abstract - The boundary problem for the two-dimensional scalar acoustic waveguide with sound speed and density Blowly varying with $x$ and arbitrary on $z$ is treated by the method of additive factorization. The equivalence between such $a$ method and the coupled modes one-way solution is proved. The resulting initial pseudodifferential problem is numerically solved with any given accuracy by the high order Pade-type approximation algorithm. The performance of the computer implementation, adopted to underwater sound propagation calculations is discussed.
\end{abstract}

\section{I - INTRODUCTION}

Many problems of waves propagation such as underwater sound propagation, long range radio waves propagation, marine seismology, light fiber propagation are of interest in case not only of layered media, but of media with properties varying in two directions. Among the mathematical procedures to predict waves fields in such circumstances the most used are the method of coupled modes $/ 1,2 /$ and the parabolic equation technique in its manifold realizations $/ 3,4 /$. In the relation following the equivalence of coupled modes or guided waves equations to the appropriate abstract parabolic equation is established and the algorithm to solve the simplified form of the last is proposed, featuring the low computation cost at the any given accuracy. II. - GUIDED WAVES EQUATIONS OFERATOR FORM.

As a model of acoustical waves propagation process we shall use the system of differential equations of type

$$
\begin{aligned}
& \left|\begin{array}{ccc}
D_{x} & 0 & -i \omega \rho \\
D_{z} & -i \omega \rho & 0 \\
-i \omega \rho^{-1} C^{-2} D_{z} & D_{x}
\end{array}\right|\left|\begin{array}{c}
p \\
v_{z} \\
v_{x}
\end{array}\right|=\left|\begin{array}{c}
{ }^{f} x \\
f_{z} \\
v^{\prime}
\end{array}\right|, \quad(x, z) \in(-\infty, \infty) \times(0, H), \\
& \gamma_{0}{ }^{P(x, 0)+v_{z}(x, 0)=0,} Y_{H}(x, H)+v_{z}(x, H)=0
\end{aligned}
$$

$p$ being acoustical pressure, $v_{x}, v_{z}$-acoustical velocity cartesian components, $f_{x}, f_{z}-$ cartesian components of external forces density, V-external volume velocity density, all of then being functions of cartesian horizontal coordinate $x$ or range and $z$ - 
vertical coordinate or depth. $D_{x}$ and $D_{z}$ are partial derivatives on $x$ and $z$, w-cyclic frequency, p-medium density, C-medium sound speed, $i^{2}=-1$, $Y_{0}$ and $Y_{H}$-acoustical admittances at upper and lower boundaries of wavequide; $H$ - the maximum depth of the waveguide, taken into account. First two equations of (1) are the Newton equations, the third is the continuity equation. Deleting from (i) the vertical component of the acoustical velocity $v_{z}$, we obtain the next operator form of (1):

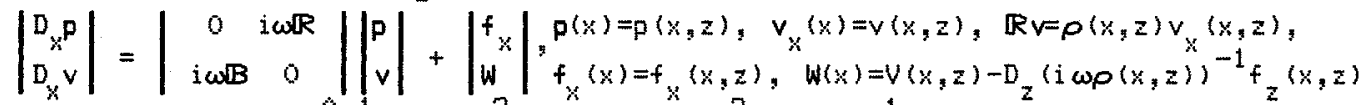

$$
\begin{aligned}
& \mathbb{B}_{p}(x)=\left(\hat{\rho}^{-1}(x, z) \mathrm{C}^{-2}(x, z)-D_{z}\left((i \omega)^{2} \rho(x, z)\right)^{-1} D_{z}\right) p(x, z), \\
& \gamma_{O} \rho(x, 0)+(i \omega \rho(x, O))^{-1} D_{2} p(x, O)=0, \quad Y_{H}\left(p(x, H)+(i \omega \rho(x, H))^{-1} D_{z} p(x, H)=0\right.
\end{aligned}
$$

Equations (2) can be seen as operator form of quided waves equations $/ 1,2 /$ assuming for the sake of simplicity $\mathbb{R}=0$-identity operator and taking into account that the operator $\mathbb{B}$ in the local normal modes $\phi(x, z)$ basis has the diagonal form

$$
\mathbb{B}(x)=\hat{\Phi}(x) \mathbb{C}^{-2}(x) \underset{\Phi}{-1}(x), \hat{\Phi}(x)=\underset{1}{\operatorname{row}}\left\{\vec{\phi}_{1}(x)\right), \mathbb{C}(x)=\underset{1}{\operatorname{diag}\left(\mathrm{C}_{1}\right\}}
$$

$c_{1}$ being the phase velocity of 1-th local normal mode, we obtain for the local normal modes amplitudes $\vec{a}(x)=\hat{\Phi}^{-1}(x) p(x), \vec{b}(x)=\hat{\Phi}^{-1}(x) \vee(x)$ :

$$
\mathrm{D}_{x} \mathrm{a}_{1}(x)-i \omega \mathrm{b}_{1}(x)=-\sum_{k} \gamma_{1 k}(x) a_{k}(x), \mathrm{D}_{x} \mathrm{~b}_{1}(x)-i \omega \mathrm{c}_{1}^{-2}(x) \mathrm{a}_{1}(x)=-\sum_{k} \gamma_{1 k}(x) \mathrm{b}_{1}(x),
$$

where $\gamma_{1 k}=\left\{\hat{\Phi}^{-1}(x)\left(D_{x} \hat{\Phi}(x)\right)\right\}_{1 k}$ are the local normal modes coupling coefficients.

III. ADDITIVE FACTORIZATION AND DNE-WAY SOLUTIONS.

In the subsequent discussion we shal use functions $f$ of linear operators $J$ accordingly to the Riss"s definition:

$$
f(T)=(2 \pi i)^{-1} \int f(\lambda)(\pi-\lambda)^{-1} d \lambda
$$

where the path of integration $\Gamma$ in the complex plain is encircling the spectre of operator $\pi$, letting him left and the operator $U$ supposed to be such, that the integral does converge.

We shall now reformulate equations (2) in the manner of Wentzel-kramerErillouin method. Let us assume, that outside of interval $x \in(a, b)$ our waveguide is layered and all sources are situated inside this interval. Introducing the new local amplitudes of waves of two opposite directions:

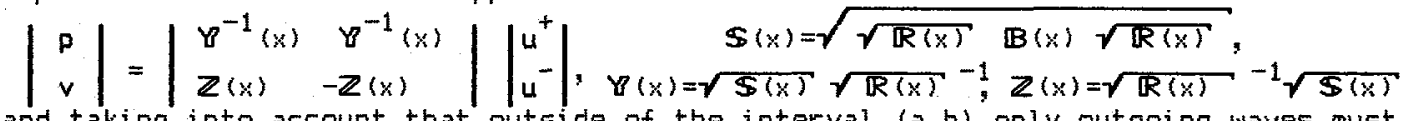

and taking into account that outside of the interval $(a, b)$ only outgoing waves must exist, we obtain by this process of additive factorization $/ 6 /$ the following system of equations:

$$
\begin{aligned}
& \left|\begin{array}{ll}
\mathbb{P} & \mathbb{U} \\
\mathbb{L} & \mathbb{Q}
\end{array}\right|\left|\begin{array}{l}
u^{+} \\
u^{-}
\end{array}\right|=\left|\begin{array}{l}
F^{+} \\
F^{-}
\end{array}\right|,\left|\begin{array}{l}
F^{+} \\
F^{-}
\end{array}\right|=1 / 2\left|\begin{array}{cc}
\gamma & \mathbb{Z}^{-1} \\
\gamma & -\mathbb{Z}^{-1}
\end{array}\right| \begin{array}{l}
f_{x} \\
W
\end{array} \mid \\
& \mathbb{P}=D_{x}-i \omega S(x)+1 / 2\left(\mathbb{Z}^{-1}(x)\left(D_{x} \mathbb{Z}(x)\right)-\mathbb{P}^{-1}(x)\left(D_{x} Y(x)\right), \quad u^{+}(a)=0\right. \\
& \mathbb{Q}=D_{x}+i \omega S(x)+1 / 2\left(\mathbb{Z}^{-1}(x)\left(D_{x} \mathbb{Z}(x)\right)-x^{-1}(x)\left(D_{x} Y(x)\right), u^{-}(b)=0\right. \\
& \mathbb{L}=\tilde{U}=-1 / 2\left(\mathbb{Z}^{-1}(x)\left(D_{x} \mathbb{Z}(x)\right)+V^{-1}(x)\left(D_{x} Y(x)\right)\right)
\end{aligned}
$$

witch can be solved by the Gauss-Zeidel iterations:

$$
u_{k}^{+}=\mathbb{P}^{-1}\left(-U_{u_{k-1}^{-}}^{-}+F^{+}\right), u_{k}^{-}=\mathbb{Q}^{-1}\left(-\mathbb{R}_{k}^{+}+F^{-}\right)
$$

converging, if $\left\|\mathbb{P}^{-1} \cup Q^{-1} \mathbb{L}\right\|<1$ for some norm $\|\cdot\|$. If $\rho$ and $\beta$ are slowly varying with $x$, then $\mathcal{U}$ and $\mathbb{L}$ are small and for well defined Cauchy"s operators $\mathbb{P}$ and $\mathbb{Q}$ the convergence of (4) is very probable. Assuming now zero field as zero order 
approximation for the first order approximation of one-way positive direction wave $u_{1}^{+}$we obtain $\hat{\mathrm{Pu}}_{1}^{+}=\mathrm{F}^{+}$, yielding in the local normal modes basis the one-way guided waves equations /5/:

$$
D_{x} c_{1}-i \omega C_{1}^{-1} c_{1}=-\sum_{k} \gamma_{1 k}\left(C_{1}+c_{k}\right)\left(2 c_{1} c_{k}\right)^{-1}+\left\langle\hat{\Phi}^{-1} F^{+}\right\}_{1}
$$

Supposing the coupling coefficients $\gamma_{1 k}$ to be fast diminishing with $|1-k|$ and therefore letting $\left(C_{1}+C_{k}\right) /\left(2 C_{1} C_{k}\right)^{-1} \cong 1$ neglecting so the difference of transmission from identity, we can obtain then in operator form the abstract parabolic equation

$$
D_{x} u_{1}^{+}-i \cos (x) u_{+}^{1}=F^{+}
$$

giving rise to the family of known PEs, according to the technique employed to approximate $\$$ and to solve the resulting approximated equation.

IV. NUMERICAL SOLUTION.

To solve (5) on the $*$ grid with step $h$ it is convenient to use the algorithm $u^{+}(x+h)=\exp (i \omega h S(x)) u^{+}(x)$ of exponential fitting type $/ 3,6,7 /$. To approximate the propagation factor operator $\mathbb{E}=\exp (i \omega h S(x)$ ) with any given accuracy we use the rational approximation: if $f(\lambda) \cong F_{n}(\lambda) / G_{m}(\lambda)\left\langle F_{n}, G_{m}\right.$ polynomials of degrees $\left.n, m\right\}$ in the vicinity of the operator's $S$ spectre then. (S) gives $f(S) \cong F_{n}(S)\left(G_{m}(S)\right)^{-1}$. We construct the appropriate approximation to exp (ih $\sqrt{\lambda}$ ) with the known Pade approximations /5/: $\exp (\lambda)=\left[M_{n}\left(\lambda^{2}\right)+\lambda N_{n}\left(\lambda^{2}\right)\right]\left[M_{n}\left(\lambda^{2}\right)-\lambda N_{n}\left(\lambda^{2}\right)\right]^{-1}+E_{n}(\lambda), \sqrt{\lambda}=F_{m}(\lambda)\left(G_{m}(\lambda)\right)^{-1}+H_{n}(\lambda)$ $M_{n}, N_{n}, F_{m}, G_{m}$ being polynomials with real coefficients, computed by recurrence, $E_{m}, H_{n}-$ approximation errors known to fade with increasing $m$ and $n$ in the complex plain without the negative half of real axes. Combining this approximations we get

$$
\begin{aligned}
\exp (i h \sqrt{\lambda)} & \left.=\int_{m}(\lambda) M_{n}\left(-h^{2} \lambda\right)+i h F_{m}(\lambda) N_{n}\left(-h^{2} \lambda\right)\right]\left(G_{m}(\lambda) M_{n}\left(-h^{2} \lambda\right)-i h F_{m}(\lambda) N_{n}\left(-h^{2} \lambda\right)\right]^{-1}= \\
& =\prod_{k=1}\left(\lambda-\mu_{k}(h)\right]\left[\lambda-\mu_{k}^{*}(h)\right]^{-1}
\end{aligned}
$$

$\mu_{k}$ being the roots of nominator in the above fraction, $\mu_{k}^{*}$ - their complex conjugated We have computed $\mu_{k}$ for some $m, n, h$. All of them are situated in the IV quadrant

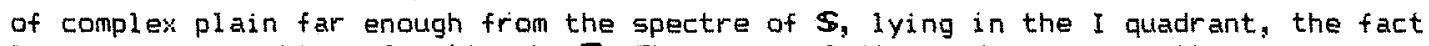
leading to stability of action by $\mathbb{E}$. The error of (6) is decreasing with $n$, $m$ increasing, but practically important is to approximate the region of. spectre, corresponding to propagating local normal modes, Iying in the vicinity of $(1,0)$ in the upper halfplain. For example, $m=4, n=4, h=4 \pi$ give for local normal modes with Brillouin angles up to $60^{\circ}$ the phase error 1 ess than $5 * 10^{-6}$ rad enabling underwater sound propagation calculations to the range of $9000 \mathrm{~km}$ with frequencies up to $100 \mathrm{~Hz}$ with absolute phase error less then $\pi / 2$.

The computer implementation of the above technique needs a discretization of vertical coordinate $z$ and an appropriate discrete approximation of $\$$. This can be done by finite-differences techniques, Galerkin method, Marchuk ${ }^{3}$ equalities method and 50 on $16 \%$. The common feature of such techniques is that the operator $\mathbb{B}$ can be approximated by the product $\hat{B}^{-1} \hat{A}$ of band matrices $\hat{B}$ and $\hat{A}$, while $\mathbb{R}$ is approximated by the diagonal matrix F, giving for E:

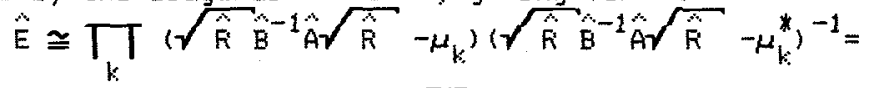

$$
\begin{aligned}
& =\prod_{k} T\left(\hat{I}-2 \operatorname{Im}\left(\mu_{k}\right) \hat{A} \sqrt{\hat{F}}-\mu_{k} \hat{B} \sqrt{\hat{F}}-1,-1 \hat{B} \sqrt{\hat{k}}-1\right)
\end{aligned}
$$

- an easy to implement algorithm, including multiplication by band matrices and solving systems of equations with such matrices. Taking now into account, that the 
rational approximation to $\mathbb{E}$ is fully determined by the set of $\mu_{k}$ " we can include into our consideration also the case of admittances $Y_{O}, Y_{H}$ depending on local normal mode phase velocity, and henceforth, on spectral parameter $\mu_{k}$, as for underlying semispace with known properties. This assumption leads to the dependence of $\hat{A}$ and $\hat{B}$ on $\mu_{k}$ due to their dependence from boundary conditions in $\mathbb{B}$ while the above form of rational approximation to $\mathbb{E}$ remains unchanged. This feature is unique to the propagation factor algorithm $(6,7)$. The another advantage of $(6,7)$ is the aggregate approximation of equation (5) and his solution resulting in lower calculations cost comparably to other known techni ques.

The three-dimensional cylindrically-symmetric problem may be treated in a similar way, substituting the propagation factors exp(t-iwsx) by zero order Hankel

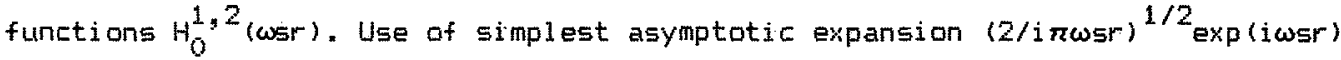
results then in the same propagation factor algorithm $(6,7)$.

The package of FofTFAN-coded routines is developed to calculate the long-range underwater sound propagation in range-depth dependent environment. Because of main interest given in this case to the sound pressure values, it uses a slightly different factorization:

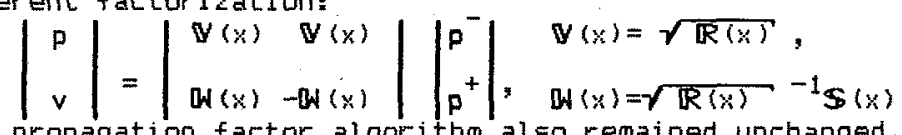

with propagation factor algorithm also remained unchanged. The performance of this package may be approximately evaluated as 50 floating points operations per one wavelength on $x$ per one node in grid on $z$ having the typical value of one fourth of the wavelength by sufficient accuracy for Briliouin angles up to $35^{\circ}$.

\section{V. - CONCLUSION.}

We have used operator notation of fluid acoustic's equations to get the pseudodifferential paraboilc equation, governing the one-way propagation of harmonic sound in range-depth dependent environment and proposed an effective numerical algorithm to solve it. In the following relations we shall include into consideration elastic media, fields with arbitrary time dependence and formulate a three-dimensional technique for horizontally slowly varying waveguides.

\section{REFERENCES}

/1/ S. Schelkunoff, Conversion of Maxwel 1"s Equations into Generalized Telegraphist"s Equations, BST. $34, N 5,795,1955$.

12/ B.Z. Katzenelenbaum, Irregular slowly varying properties waveguides theory, Moscow, 1961.

13/ F. D. Tappert, The Farabolic Approximation Method, in Wave Fropagation and Underwater Acoustics, edited by J.B.Keller and J.S.Papadakis, Lecture Notes in Fhysics, Vol. 70, Springer, New-York, 1977.

14/ A. Eamberger, B. Engquist, L. Halpern and P.Joly, Higher Order Paraxial Wave Equation Approximation in Heterogeneous Media, SIAM. J. Appl. Math., 48, 129-154, (1988)

/5/ F.Riesz, E.Sz-Nagy, Lecons d"analyse fonctionnel, Akadeniai Kiado, Budapest, 1972

/t/ I.Babuska,M.Frager, E.Vitasek, Numerical processes in differential equations, Fraha. 1966.

17/ Y.L.Luke, Mathematical functions and their approximations, Acadenic Fress; 1975

/8/ C.V.Aviloff, Calculation of wavequides harmonic sound fields in a corrected wide-angle parabolic approximationg in:Al1-union's symposium on waves and diffraction-85 proceedings, Tbilissi, 1985.

19/ C.V.Aviloff, Dne-way propagation approximation in ocean acoustics, in: Acoustics of ocean medium, edited by L.M.Brekhovskikh, I.B. Andreeva, Moscow, Nallia, 1989. 